\title{
BMJ Open INtrinsic Capacity and its RElAtionship With Life-SpacE Mobility (INCREASE): a cross-sectional study of community- dwelling older adults in Singapore
}

\author{
Jia Qi Lee (D , , Yew Yoong Ding, ${ }^{2,3}$ Aisyah Latib, ${ }^{4}$ Laura Tay, ${ }^{3,5}$ Yee Sien Ng ${ }^{1,3,6}$
}

To cite: Lee JQ, Ding YY, Latib A, et al. INtrinsic Capacity and its REIAtionship With LifeSpacE Mobility (INCREASE): a cross-sectional study of community-dwelling older adults in Singapore. BMJ Open 2021;11:e054705. doi:10.1136/ bmjopen-2021-054705

- Prepublication history and additional supplemental material for this paper are available online. To view these files, please visit the journal online (http://dx.doi.org/10.1136/ bmjopen-2021-054705).

LT and YSN contributed equally.

Received 21 June 2021 Accepted 29 November 2021

Check for updates

(C) Author(s) (or their employer(s)) 2021. Re-use permitted under CC BY-NC. No commercial re-use. See rights and permissions. Published by BMJ.

${ }^{1}$ Duke-NUS Medical School, Singapore

${ }^{2}$ Geriatric Medicine, Tan Tock Seng Hospital, Singapore

${ }^{3}$ Geriatric Education \& Research Institute, Singapore

${ }^{4}$ Singhealth Regional Health System, Singapore

${ }^{5}$ General Medicine, Sengkang General Hospital, Singapore

${ }^{6}$ Rehabilitation Medicine,

Singapore General Hospital, Singapore

Correspondence to

Jia Qi Lee;

jiaqi.lee@u.duke.nus.edu

\section{ABSTRACT}

Objectives To examine the association of intrinsic capacity (IC) with life-space mobility (LSM) among community-dwelling older adults and to determine whether age and gender modify this relationship. Design Cross-sectional study.

Setting Public housing blocks, senior activity centres and community centres in the Northeastern region of Singapore.

Participants 751 community-dwelling older adults aged $\geq 55$ years old and able to ambulate independently with or without walking aid.

Primary and secondary outcome measures IC and LSM. Standardised IC factor scores were calculated through confirmatory factor analysis using variables representing the five IC domains cognition, locomotion, sensory, vitality and psychological. LSM was measured using the University of Alabama at Birmingham Study of Aging Life-Space Assessment instrument. Association of IC with LSM and its effect modification by age and gender were examined with regression analyses.

Results The participants had a mean age of 67.6 and mean LSM score of 88.6. IC showed a positive and significant association with LSM $(\beta=6.33 ; 95 \% \mathrm{Cl}=4.94$ to 7.72 ) and the effect remained significant even after controlling for potential confounders $(\beta=4.76$; $95 \%$ $\mathrm{Cl}=3.22$ to 6.29 ), with $\mathrm{p}<0.001$ for both. Age and gender did not demonstrate significant modification on this relationship.

Conclusions Our findings support the empirical rigour of the International Classification of Functioning, Disability and Health framework, which suggests that IC influences the extent to which a person participates in the community. Our findings also provide guidance for healthcare providers who aim to enhance LSM and promote healthy ageing in older adults.

\section{INTRODUCTION}

As population ageing becomes increasingly prevalent worldwide, many countries are witnessing an epidemiological transition characterised by the rising burden of chronic and degenerative diseases. ${ }^{1}$ In addition to the need for long-term healthcare, older adults also face health-related problems such as malnutrition, depression and isolation. ${ }^{23}$
Strengths and limitations of this study

- Multiple linear regression controlling for various confounders including demographic, economic and social factors was used to demonstrate independent association of intrinsic capacity (IC) with life-space mobility (LSM).

- IC factor scores for each participant were calculated from a number of self-reported and observed variables using structural equation modelling and used in our analyses.

- The cross-sectional design of the study limits the determination of causality and directionality in the relationship of IC and LSM.

- The study sample consisted of relatively high functioning older adults with high Activities of Daily Living (ADL) and Instrumental ADL scores, which limits generalisability of our findings to other populations.

Unfortunately, current healthcare systems are designed largely to provide fragmented and episodic short-term care, which may not adequately address the complex health needs of older adults. ${ }^{4}$ Hence, there is a need to work towards a more person-centred model of care that follows older adults through their life course and that addresses not just their biological, but also their psychological and social needs. ${ }^{56}$

To facilitate this paradigm shift, WHO proposed a new conceptual model for 'Healthy ageing' in 2015, in which healthy ageing is defined as 'the process of developing and maintaining the functional ability that enables well-being in older age'. ${ }^{4}$ As part of this model, intrinsic capacity (IC), defined as 'the composite of all physical and mental capacities of the person', was introduced as a multidimensional indicator that can be used as a measure of health in older people over time. $^{4}$

IC is conceptualised as having five domains, namely cognition, locomotion, sensory, vitality and psychological. ${ }^{7}$ It has been shown 
to be reliably estimated from a range of frequently collected biomarkers and self-reported measures representing these five domains. ${ }^{8} 9$ IC declines have been shown to predict disease manifestations and decrements in Activities of Daily Living (ADL) and Instrumental ADL (IADL). ${ }^{810}$ As such, IC levels of older adults can be tracked over time to allow early identification of abnormal deviations and timely interventions to prevent deterioration. ${ }^{710}$ Furthermore, compared with crude risk assessment measures like chronological age, IC provides valuable information about one's physiological reserves, which can help guide healthcare providers in identifying the populations to target for interventions. ${ }^{112}$ The multi-domain nature of IC also encourages integration of services and formulation of multi-pronged, multidisciplinary strategies, which are likely to be more effective in addressing the complex needs of older adults. ${ }^{10} 12$ Moreover, unlike many traditional measures of health, IC carries a positive connotation by focusing on the preserved capacities of an individual, rather than their deficits and limitations. ${ }^{7}$

Taken together, IC can potentially be adopted in clinical practice as a useful tool to monitor and assess older adults' functional health status over time. At present, IC is still largely a theoretical construct as there is lack of a standardised metric and guidelines for its operationalisation in clinical and research settings. ${ }^{79}$ There have been few empirical studies elucidating the association and predictive value of IC with ADLs, IADLs and adverse health events including falls, hospitalisation and mortality in older adults. ${ }^{8}{ }^{13-15}$ However, according to the 2001 International Classification of Functioning, Disability and Health (ICF) framework (see online supplemental figure 1), apart from activities (measured by ADLs and IADLs), participation, defined as one's involvement in a life situation and engagement in the community, is another important aspect of functioning. ${ }^{16}$ Based on the framework, one's body functions (or IC) influences an individual's ability to be and to do what they have reason to value, which directly affects the extent to which they participate in the community. ${ }^{4716}$ The effect of IC on participation is in turn modified by contextual factors (environment and personal) and health conditions. However, the association between IC and participation is yet to be established empirically.

An individual's participation in the community can in turn be measured by his/her life-space mobility (LSM), which refers to the ability to move or travel in one's daily life, taking into account the frequency of movement and degree of independence. ${ }^{17} 18$ Constricted LSM has been shown to be associated with negative outcomes such as increased risk of Alzheimer's disease and cognitive decline, frailty, decreased quality of life and even mortality. ${ }^{19-22}$ While many studies have demonstrated the effect of environmental barriers like poor street conditions on LSM in older adults, the association between IC and participation is much less established. ${ }^{2324}$ Elucidation of this association would provide valuable information for healthcare providers when designing interventions that promote healthy ageing.

\section{Aims and hypotheses}

Using data from an ongoing community-based study, the Individual Physical Proficiency Test for Seniors (IPPTS), we aim to: (1) examine the association of IC and the five domains with LSM and (2) investigate if age and gender modify the relationship between IC and LSM in community-dwelling older adults in Singapore. We hypothesise that IC is positively associated with LSM, as suggested by the ICF framework. We also hypothesise that increasing age and male gender attenuate the effect of IC on LSM, since older age has been associated with increased fear of falling and decreased confidence leading to restriction in community participation, while male older adults have been shown to participate less in community activities and have smaller social networks than their female counterparts. ${ }^{25-28}$

\section{MATERIALS AND METHODS Study description}

The IPPT-S is an ongoing community-based frailty screening programme that aims to promote fitness and prevent or delay frailty progression in older adults. ${ }^{29}$ Data from June 2018 to October 2019 were used for analysis in this study. Community-dwelling older adults residing in the Northeastern region of Singapore were invited through senior activity centres and resident committees. Inclusion criteria for participants include (1) age $\geq 55$ years old, (2) community-dwelling, and (3) ability to ambulate independently (with or without walking aid). Individuals who live in residential care facilities were excluded. The assessment consisted of two components, a multidomain questionnaire and the short physical performance battery (SPPB). ${ }^{29} 30$

\section{Patient and public involvement}

No patients or members of the public were involved in the development of the research question, study design or interpretation of the data.

\section{Measures of IC}

Variables representing the five domains of IC, namely cognition, locomotion, sensory, vitality and psychological were included in the measurement of IC. All data had been collected by trained members of the study team, including research assistants, physiotherapists and nutritionist.

\section{Measures of locomotion domain}

\section{SPPB Total Score}

The SPPB consists of three tests (balance, chair stand and gait speed). ${ }^{29}$ Tests of static balance included side-by-side, semitandem and full-tandem standing and time taken to remain in position was measured. For chair stand, time taken to complete five chair stands was recorded. 
Gait speed was measured by duration of walking $10 \mathrm{~m}$ at normal pace.

\section{Timed up and go}

Timed up and go (TUG) is a test for dynamic balance and functional mobility. ${ }^{31}$ Time taken to rise from a chair, walk $3 \mathrm{~m}$, go round a cone and return to the seat was recorded.

\section{5 times sit-to-stand}

5 times sit-to-stand (5TSTS) measures lower body strength. ${ }^{32}$ Participants stood from a sitting position as fast as they could with arms folded across their chest, and time taken to complete five repeats were measured.

\section{Measures of psychological domain} Geriatric Depression Scale

The 15-item Geriatric Depression Scale (GDS) consists of yes/no questions pertaining to depressive symptoms and a score of $\geq 5$ indicates depression. ${ }^{33}$

\section{EuroQol-5 Dimensions (EQ-5D) anxiety/depression}

Participants were scored from 0 to 4 based on the severity of anxiety/depression they felt, with 0 being 'none' and 4 being 'extreme'.

\section{Measures of cognition domain Cognitive}

The Chinese Mini Mental State Examination (CMMSE) ${ }^{35}$ consists of 18 items and measures three kinds of cognitive functions: (1) Memory-measured by delayed word recall test, (2) Orientation-measured by summating the scores on eight items that test participants' orientation to time and place, and (3) Executive function-measured by summating the scores on five tasks: repeat the following phrase, three-stage command, say a sentence, read and obey what is written on the paper and copy this drawing.

\section{Measures of sensory domain \\ Sensory}

Sensory functions were assessed by self-reported absence or presence of hearing and/or visual impairments.

\section{Measures of vitality domain \\ Exhaustion}

Participants rated how frequently the statement 'I felt that everything I did was an effort' applied to them over the past week on a 4-point Likert scale, with 0 being 'Rarely or none of the time (less than 1 day)' to 3 being 'Most or all of the time (5-7 days)' ${ }^{36}$

\section{Mini Nutritional Assessment-Short Form}

The Mini Nutritional Assessment-Short Form (MNA$\mathrm{SF}$ ) identifies geriatric patients who are malnourished or at risk of malnutrition. ${ }^{37}$ It also screens for dementia, depression, mobility, acute disease and psychological stress. A score of $<8$ suggests malnutrition, 8-11 suggests risk of malnutrition, while 12-14 suggests normal nutritional status.

\section{Grip strength}

Grip strength was measured with a JAMAR Plus Hand Dynamometer (Sammons Preston, Bolingbrook, Illinois, USA). ${ }^{38}$ Two trials were conducted for each hand and the higher reading was used for analysis.

\section{LSM as a measure of participation}

LSM was measured using the University of Alabama at Birmingham Study of Aging Life-Space Assessment (UABLSA) instrument. ${ }^{18}$ The UAB-LSA defines five life-space levels: (1) rooms of your home other than the bedroom, (2) an area outside your home such as your porch, deck or patio, hallway (of an apartment building) or garage, in your own yard or driveway, (3) places in the neighbourhood, (4) places outside of your neighbourhood, but within your town and (5) places outside your town. For each life-space level, participants reported their frequency of attainment and degree of dependence. The frequency of attainment was scored from 0 (never) to 4 (daily), while the degree of dependence was scored 1, 1.5 or 2 representing 'personal assistance required', 'walking device required' and 'independent', respectively. A final composite score that ranges from 0 (completely homebound) to 120 (maximum mobility) was calculated. The minimum LSM score needed to be considered high participation is 60 , since a person must fulfil at least lifespace levels 1 and 2 to attain this score. ${ }^{39}$

\section{Other measures included in the study}

In addition to demographic information (age, gender, ethnicity), participants were asked a number of questions regarding social determinants, including socioeconomic status (highest educational level, housing type, employment status), lifestyle (smoking, alcohol) and social support and network. ${ }^{29}$ Frailty, functional status and chronic medical conditions were also included as descriptive measures of the study sample. The five-item FRAIL (Fatigue, Resistance, Ambulation, Illnesses and Loss of weight) assessment was used to measure frailty and categorise participants as frail (score of 3-5), prefrail (1-2) or robust (0) ${ }^{40}$ Functioning in ADL and IADL was assessed using the Barthel Index ${ }^{41}$ and Lawton and Brody's scale, ${ }^{42}$ respectively. Self-reported presence or absence of eleven chronic diseases was recorded.

\section{Statistical analysis}

An exploratory factor analysis (EFA) was performed to identify the underlying factor structure of the IC construct using available data from our study population. Thirteen variables, SPPB, TUG, 5TSTS, GDS, EuroQol-5 Dimensions (EQ-5D) anxiety/depression, Memory, Executive, Orientation, Hearing, Vision, Exhaustion, MNA-SF and Grip strength, were included in the EFA based on empirical evidence $^{89}$ and their theoretical associations with the five IC domains. Seven of the variables, TUG, 5TSTS, GDS, EQ-5D anxiety/depression, Hearing, Vision and Exhaustion, were reverse-coded such that higher values represented better performance. Eigenvalues were 
used to identify the number of factors to retain and a cutoff of $\geq 0.4$ factor loading was used to select the items to include in the model. The identified factor structure was further tested with confirmatory factor analysis (CFA). The CFA model was validated by assessing its fit with data using several goodness-of-fit indices: Root Mean Square Error of Approximation (RMSEA), Comparative Fit Index (CFI) and Tucker-Lewis Index (TLI). The criteria for good fit with data are RMSEA $\leq 0.8$ and CFI and TLI values of $\geq 0.9 .^{43}$ Standardised factor scores for IC were calculated and applied in subsequent analyses.

Age was centred by subtracting all values by 65 , such that 0 represents 65 years old and -10 represents 55 years old. The centred age variable was used in subsequent analyses. Simple linear regression was performed for each of the baseline demographic, economic and social characteristics with IC and LSM to identify potential confounders. Sequential regression analyses of LSM on IC were then performed: (1) the first model included LSM as dependent variable and IC as independent variable, (2) the second model was the first model with age, gender, education, housing, disposable income, employment, living alone, attendance in community/religious activities, having a confidant, contact with relatives/ friends, helping others added as covariates and (3) the third model was the second model with addition of IC $\mathrm{x}$ age and IC $\mathrm{x}$ gender interactions. Chronic conditions were not included as covariates in our regression analyses as they are closely related to IC and separating their effects is not likely to be meaningful in answering the research questions posed in this study.

Standardised factor scores for the five subfactors were also calculated and applied in regression analyses to examine the association between the individual IC domains and LSM. All statistical analyses were performed using Stata/SE V.16.1 and R V.4.0.3.

\section{RESULTS}

\section{Sample characteristics}

Of 761 participants from the IPPT-S study, 751 were included in our analyses after removal of individuals with missing data in LSM. Age, gender and frailty status of the excluded participants were similar to the cohort included for analysis. Table 1 summarises the baseline sample characteristics. The mean age of participants was 67.6 (SD 7.03), with most participants being female $(71.8 \%$, $\mathrm{N}=539$ ). $85.8 \%$ of participants were ethnically Chinese. Malays and Indians/other ethnicities constituted 9.72\% and $4.53 \%$ of the participants respectively, which are slightly lower than in the 2020 national statistics $(15 \%$ and $9.1 \%$, respectively). ${ }^{44}$ The mean total LSM score was 88.6 (SD 20.4) out of the maximum score of 120 , with the lowest score being 16 and highest $120.92 .3 \%$ of the participants $(\mathrm{N}=693)$ attained a score of at least 60 and were regarded to have high participation, while the remaining $7.72 \%(\mathrm{~N}=58)$ have low participation with LSM score $<60$.
EFA, CFA and model fit

Summary statistics of the 13 variables used in the EFA are presented in table 2 . The variables represented the five domains of IC: locomotion-SPPB, TUG, 5TSTS; psychological-GDS, EQ-5D anxiety/depression; cognitionMemory, Orientation, Executive; sensory-Hearing, Vision; vitality-Exhaustion, MNA-SF and Grip strength.

Table 3 shows the results of the EFA. The EFA suggested a five-factor model, with Eigenvalues ranging from 1.06646 to 2.62684 . The five factors corresponded to the five IC domains, with items representing the domains locomotion, psychological, cognition, sensory and vitality loading onto factors $1-5$, respectively. An exception lies in factor 2 in which there was cross-loading of psychological and vitality items-GDS and EQ-5D anxiety/depression (which are psychological measures) had factor loadings of 0.7881 and 0.7116, while Exhaustion and MNA-SF (which are vitality measures) had factor loadings of 0.4982 and 0.5563 , respectively. However, in general, all items loaded well onto their respective factors, with factor loadings greater than or equal to 0.4 (range $0.4364-0.9049$ ).

The EFA results were used to guide development of the CFA model. Variables Exhaustion and MNA-SF were mapped onto vitality domain despite the EFA findings due to consideration of their conceptual association with the IC vitality domain. Grip strength was removed from the model as an initial CFA iteration revealed poor factor loading of 0.105. The eventual CFA model was a second-order model with one general factor (IC) and five subfactors (locomotion, psychological, cognition, sensory and vitality) (figure 1). The model achieved good fit with data: $\chi^{2}=136.7(\mathrm{df}=49)$, RMSEA $=0.049(90 \%$ CI 0.039 to 0.059 ), CFI $=0.954$ and TLI $=0.937$. Ten of the 12 items had factor loadings greater than 0.4 (range $0.408-0.996$ ). The remaining two items, Hearing and Memory, had factor loadings of 0.326 and 0.355 , respectively, which are still in acceptable range. Of note, there is substantial variability in the loadings of the subfactors onto the general factor (IC), where cognition has the lowest factor loading of 0.155 while vitality has the highest factor loading of 0.979 among the five subfactors. This suggests that the five domains were not equally represented in the IC construct based on this model.

\section{Associations between IC and LSM}

Based on the second-order CFA model, standardised IC factor scores were calculated for each participant and used in subsequent regression analyses. IC was shown to have a statistically significant positive association with LSM ( $\beta=6.33 ; 95 \%$ CI $=4.94$ to $7.72 ; p<0.001)$ in a simple linear regression model (table 4 ). In other words, $1 \mathrm{SD}$ increase in factor score for IC increases LSM by approximately six units. Given that three units of LSM represents the minimal clinically important difference (MCID) for $\mathrm{LSM}^{45}$ this effect is also clinically significant. Potential confounders were identified as variables that showed statistically significant $(p<0.05)$ association with IC and LSM. Multiple linear regression analysis which included 
Table 1 Summary of baseline sample characteristics



${ }^{*}$ Number of observations vary across variables due to missing data ( $\left.\mathrm{N}=734-751\right)$.

$\mathrm{BI}$, Barthel Index; BMI, body mass index; FRAIL, fatigue, resistance, ambulation, illness and loss of weight; IADL, instrumental activities of daily living; LSM, life-space mobility. 
Table 2 Descriptive statistics of measures of intrinsic capacity



*Number of observations vary across variables due to missing data $(\mathrm{N}=721-749)$.

CMMSE, Chinese Mini Mental State Examination; EQ5D, EuroQol-5 Dimensions; GDS, Geriatric Depression Scale; IC, intrinsic capacity; LSM, life-space mobility; MNA-SF, Mini Nutritional Assessment-Short Form; SPPB, Short Physical Performance Battery; 5 TSTS, 5 Times Sit-ToStand; TUG, Timed Up and Go.

demographic (age, gender, education), economic (housing, disposable income, employment) and social variables (living alone, attendance in community/religious activities, having a confidant, contact with relatives/friends, helping others) revealed that IC remained independently associated with LSM $(\beta=4.76 ; 95 \% \mathrm{CI}=3.22$ to $6.29 ; \mathrm{p}<0.001)$. Thus, controlling for relevant demographic, economic and social factors, 1 SD increase in factor score for IC increases LSM by almost five units. This still represents a clinically significant effect. In the multiple linear regression model that included interaction terms IC $\mathrm{x}$ age and IC $\mathrm{x}$ gender, age and gender did not show significant modification on the effect of IC on LSM ( $\beta=0.082 ; 95 \%$ CI $=-0.114$ to $0.278 ; \mathrm{p}=0.412$ and $\beta=-0.130 ; 95 \% \mathrm{CI}=-2.97$ to $2.71 ; \mathrm{p}=0.928$, respectively). Details of the above regression models are presented in online supplemental table 1.

\section{Associations between IC domains and LSM}

All five IC domains showed positive and significant association with LSM ( $\beta=4.76$ to $\beta=7.52)$, which remained significant even after controlling for confounders $(\beta=2.55$ to $\beta=5.87$; table 4 ). Among them, locomotion had the strongest association with LSM $(\beta=7.52$ before and $\beta=5.87$ after controlling for confounders). This means that $1 \mathrm{SD}$ increase in locomotion factor score increases LSM score by 5.87 units after accounting for confounding factors, and this effect is clinically significant $(\beta=5.87$; $>$ MCID of 
Table 3 Exploratory factor analysis of intrinsic capacity items $(\mathrm{N}=751)$

\begin{tabular}{|c|c|c|c|c|c|c|}
\hline \multirow[b]{2}{*}{ IC domain } & \multirow[b]{2}{*}{ Items } & \multicolumn{5}{|l|}{ Factor } \\
\hline & & 1 & 2 & 3 & 4 & 5 \\
\hline \multirow[t]{3}{*}{ Locomotion } & SPPB & 0.9018 & 0.0160 & 0.0967 & 0.0034 & 0.0304 \\
\hline & TUG & 0.8462 & 0.0352 & 0.1474 & 0.0280 & 0.0347 \\
\hline & 5TSTS & 0.9049 & 0.0347 & 0.0265 & 0.0288 & 0.0430 \\
\hline \multirow[t]{2}{*}{ Psychological } & GDS & 0.1175 & 0.7881 & 0.0568 & 0.1538 & -0.0775 \\
\hline & EQ-5D anxiety/depression & -0.0604 & 0.7116 & -0.0393 & -0.0412 & 0.0209 \\
\hline \multirow[t]{3}{*}{ Cognition } & Memory & 0.0677 & 0.1373 & 0.4571 & -0.0535 & -0.5171 \\
\hline & Orientation & 0.1356 & 0.0008 & 0.7941 & 0.0023 & 0.0068 \\
\hline & Executive & 0.2728 & -0.0252 & 0.6374 & 0.0402 & 0.0138 \\
\hline \multirow[t]{2}{*}{ Sensory } & Hearing & -0.0563 & 0.0693 & 0.1624 & 0.7287 & 0.2789 \\
\hline & Vision & 0.1128 & 0.0954 & -0.1270 & 0.7608 & -0.2387 \\
\hline \multirow[t]{6}{*}{ Vitality } & Exhaustion & 0.1954 & 0.4982 & -0.2058 & 0.0698 & -0.1601 \\
\hline & MNA-SF & -0.0632 & 0.5563 & 0.0835 & 0.0872 & 0.4364 \\
\hline & Grip strength & 0.3251 & -0.0120 & 0.0427 & -0.0595 & 0.6614 \\
\hline & Eigenvalue & 2.62684 & 1.72141 & 1.37600 & 1.15724 & 1.06646 \\
\hline & $\%$ of total variance & 20.21 & 13.24 & 10.58 & 8.90 & 8.20 \\
\hline & Cumulative \% & & & & & 61.13 \\
\hline
\end{tabular}

Extraction method: principal component factors. Rotation method: orthogonal varimax without Kaiser normalisation. Loadings larger than 0.40 are highlighted in bold.

EQ5D, EuroQol-5 Dimensions; GDS, Geriatric Depression Scale; IC, intrinsic capacity; MNA-SF, Mini Nutritional Assessment-Short Form; SPPB, Short Physical Performance Battery; 5TSTS, 5 Times Sit-To-Stand; TUG, Timed Up and Go.

three). On the other hand, cognition showed the weakest effect on LSM $(\beta=4.76)$ and this was non-clinically significant after controlling for confounders $(\beta=2.55$; $<$ MCID of three).

\section{DISCUSSION}

IC and LSM are two concepts that are of increasing interest in the field of population health. To our knowledge, this is the first study to consider the two concepts together and examine their association.

We demonstrated that IC has a positive and clinically significant effect on LSM, even after accounting for age, gender and other demographic and socioeconomic factors. This aligns with the ICF theoretical framework, which suggests that IC contributes to older adults' ability to participate in the community. ${ }^{16}$ Our findings also corroborate with prior empirical evidence which demonstrated that older adults with frailty, a state of vulnerability to adverse health outcomes characterised by a decline in IC, have more restricted LSM compared with robust older adults. ${ }^{46}$ Taken together, this suggests that intrinsic factors and physiological reserves of older adults have a role in determining how active they are in the community. However, notwithstanding the positive association between IC and LSM, it is important to consider that the ability of older adults to age well and correspondingly their life-space can be influenced by the physical environment serving as a barrier or facilitator and its interaction with IC. ${ }^{47}$

In addition, even in this cohort of relatively well and functionally independent older adults, as reflected by the overall high ADL and IADL scores, and only $1.2 \%$ being overtly frail, we have observed a significant positive association between IC and LSM. This further supports the concept of IC in monitoring overall health status of older people before they experience significant losses in functional ability and LSM.

Contrary to our initial hypothesis, our results showed that the magnitude of effect of IC on LSM does not vary according to age or gender. This suggests that effect of IC on LSM does not need to be considered in age-specific or gender-specific terms. The implication of this is that measures seeking to improve LSM by enhancing IC would apply to older adults across age and gender.

We also found that out of the five IC domains, locomotion has the strongest effect on LSM. This can be attributed to the fact that poorer physical performance and lower limb strength reduce one's ability to walk safely, which in turn deters a person from travelling long distances or going outdoors as frequently. ${ }^{48}$ Although older adults are able to compensate for their physical deficits by using assistive devices or having personal assistance, their reliance on assistance implies that they have more limited autonomy over when and where they travel, which consequently results in more restricted LSM. ${ }^{48}$ 


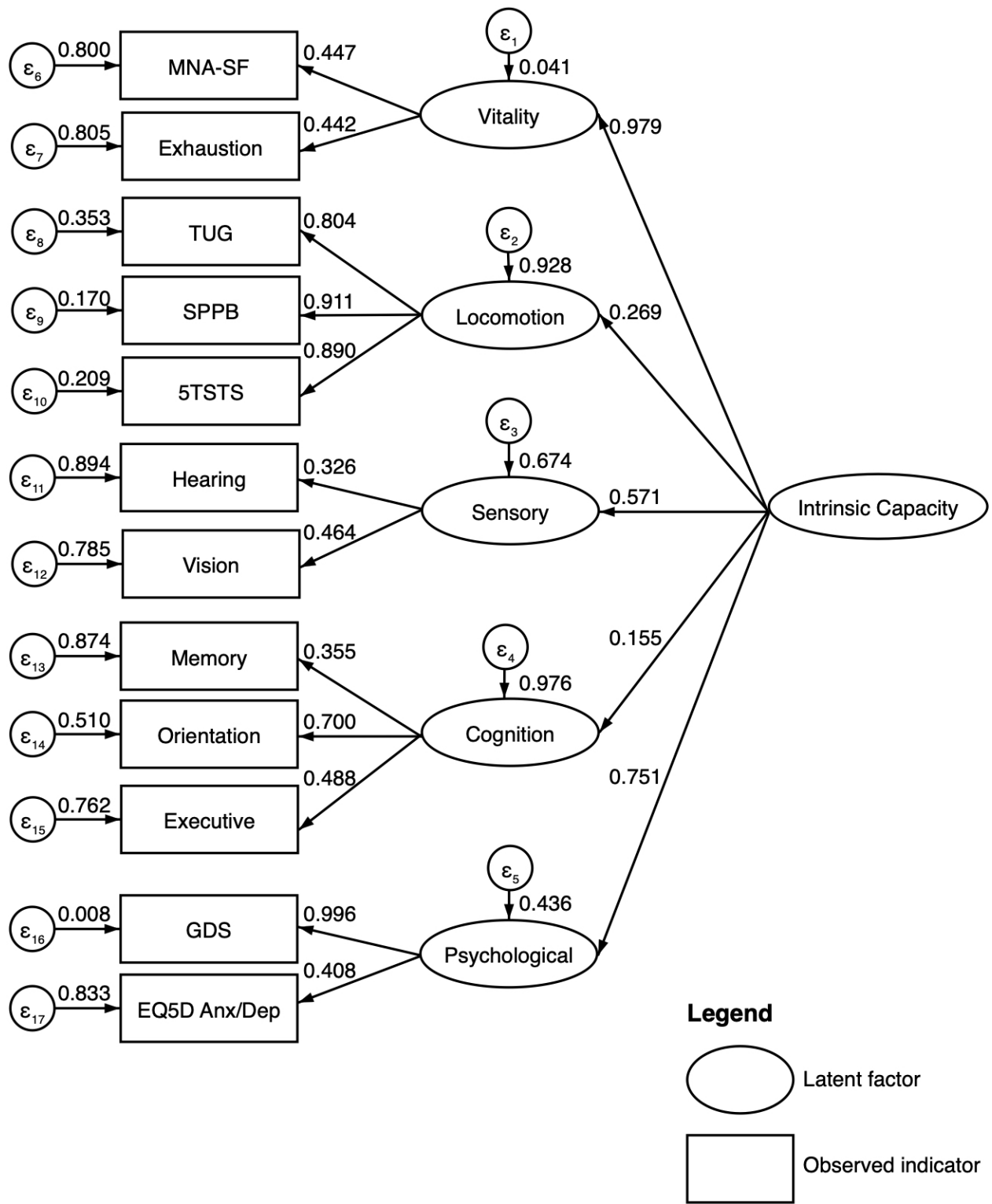

Figure 1 Second-order confirmatory factor analysis (CFA) model of intrinsic capacity. 5TSTS, 5 times Sit-To-Stand; EQ-5D Anx/Dep, EuroQol-5 dimensions Anxiety/Depression; GDS, Geriatric Depression Scale; MNA-SF, mini nutritional AssessmentShort form; SPPB, short physical performance battery; TUG, Timed Up And Go.

On the other hand, cognition was shown to have the weakest, although still statistically significant, association with LSM among the five domains. Unlike locomotion, there have been mixed reports regarding the relationship between cognitive function and life-space, with some studies demonstrating significant association of higher cognitive function with increased mobility, ${ }^{18} 49$ and others reporting otherwise..$^{50}$ A larger life-space arguably connotes increased environmental complexity, and thus necessitates higher-order cognitive functions like planning, decision-making and judgement to successfully navigate through the environment. ${ }^{49}$ However, having intact cognitive function may not necessarily translate into greater LSM. This has been attributed to the notion of self-induced dependence, where older persons behave in accordance with others' expectations of them instead of performing at their true ability level. ${ }^{49}$ Many negative stereotypes of ageing about the elderly continue to prevail in many societies, for instance, the perception of cognitive impairment and 'senility' as an inevitable consequence of ageing. As a result, such societal impressions may insidiously affect older adults' perceptions of their own abilities and cause them to limit their mobility below what they are capable of achieving.

The weak association of cognition with LSM could also be due to the fact that CMMSE has a ceiling effect on individuals with higher educational attainment, whereby highly functioning individuals with very early cognitive impairment may still perform within the normal range, that is, scoring $\geq 21$ out of $28 .{ }^{53}$ However, there is a lack of more granular psychometric measures of cognition to circumvent this problem. 
Table 4 Regression analyses of intrinsic capacity and its domains on LSM

\begin{tabular}{|c|c|c|c|c|}
\hline \multirow[b]{3}{*}{ Predictor } & \multicolumn{4}{|l|}{ Model } \\
\hline & \multicolumn{2}{|l|}{1} & \multicolumn{2}{|l|}{2} \\
\hline & $\beta$ (SE) & $95 \% \mathrm{Cl}$ & $\beta$ (SE) & $95 \% \mathrm{Cl}$ \\
\hline Locomotion & $7.52(0.692)^{\star}$ & (6.17 to 8.88 ) & $5.87(0.769)^{*}$ & (4.36 to 7.38 ) \\
\hline Psychological & $4.98(0.722)^{\star}$ & (3.56 to 6.39 ) & $3.50(0.780)^{*}$ & (1.97 to 5.03 ) \\
\hline Sensory & $5.69(0.715)^{\star}$ & (4.29 to 7.09 ) & $3.70(0.750)^{*}$ & (2.23 to 5.17 ) \\
\hline Vitality & $6.29(0.708)^{\star}$ & (4.90 to 7.68 ) & $4.74(0.781)^{\star}$ & (3.20 to 6.27 ) \\
\hline
\end{tabular}

Model 1: Univariate regression model with LSM as the outcome variable; Model 2: Multivariable regression model with LSM as the outcome variable and demographic (age, gender, education), economic (housing, disposable income, employment) and social (living alone, attendance in community/religious activities, having a confidant, contact with relatives/friends and helping others) variables added as covariates.

${ }^{*} \mathrm{P} \leq 0.001$.

IC, intrinsic capacity; LSM, life-space mobility.

\section{Strengths and limitations of study}

This study demonstrated that multiple observable and measurable variables can be aggregated into a composite IC score using factor analysis. Given that there is currently no standardised tool/instrument for the quantitative measure of IC, this study thus contributes to the growing literature that supports the use of this construct in the assessment of older adults and also aids in the development of tools/instruments that enable the clinical application of IC.

Furthermore, our results suggest that IC is a potential target for interventions that aim to enhance LSM and promote healthy ageing in older adults. Such interventions are likely to adopt a more functions-based and proactive approach by engaging and empowering seniors to be involved in improving their own health. Interventions are also likely to be more personalised and tailored to individual needs, priorities and values. Moreover, while interventions targeting IC and those addressing environmental factors such as building infrastructure or social support are clearly complementary in enhancing participation, the former may be less costly to implement.

The study also has limitations to be acknowledged. Given that the analyses were cross-sectional by design, causal inferences and directionality could not be determined. As such, the associations should be examined further using longitudinal data. A recent study on longitudinal ageing profiling identified a set of biomarkers including proteins, cytokines and clinical laboratory values, as well as subjective measures like physical activity and dietary habits, which trajectories can be monitored over time to provide insight into individuals' ageing patterns. ${ }^{54}$ Future studies involving the longitudinal measurement of IC could thus potentially adopt these measures. A second limitation is that the study sample consisted of relatively high functioning older adults. Moreover, individuals with mobility limitations (inability to ambulate at least $4 \mathrm{~m}$ independently) were excluded. As a result, the associations of IC and its domains with LSM in this study may be underestimated. This also limits the generalisability of our results to other populations. Lastly, the use of self-reporting instrument UAB-LSA to assess LSM may result in subjective and inaccurate measurements due to recall bias, particularly in older adults with cognitive impairment. However, the UAB-LSA instrument has been shown to correlate well with objective data from tracking devices. ${ }^{55}$ In addition, the sensory domain was measured using self-reported questionnaire with binary yes/no questions without delineation of the severity of impairment. Objective measures of visual acuity and audiometry could have been adopted for more accurate assessments.

\section{CONCLUSION}

In conclusion, this study demonstrates that IC has a positive effect on LSM which is statistically and clinically significant. Age and gender do not significantly modify the relationship between IC and LSM. These findings provide valuable information for healthcare providers who aim to enhance LSM and promote healthy ageing in older adults.

Acknowledgements We acknowledge the IPPT-S study team for their efforts in the administration of the study and data acquisition. We thank the study participants, staff of the Senior Activity Centres, and Resident Committees in the northeast region of Singapore for their logistical and manpower support. We would also like to extend our gratitude to Dr Fan Qiao from the Centre for Quantitative Medicine, Duke-NUS Medical School for her guidance on our statistical work.

Contributors JQL, YYD and YSN conceived of the research and were involved in the conceptualisation and design of the study. JQL undertook the analyses and was responsible for the final drafting of the paper. YYD oversaw the analyses and contributed to the drafting of the paper. YSN and LT are principal investigators of the IPPT-S study from which data were used. AL was involved in data acquisition and management. All authors reviewed the accuracy and integrity of the work. YSN is the guarantor of the work and is responsible for the overall content.

Funding This study is funded by the Singapore Ministry of Health's National Medical Research Council under the Fellowship Programme by SingHealth Regional Health System, Population-based, Unified, Learning System for Enhanced and Sustainable (PULSES) Health Centre Grant (NMRC/CG/C027/2017_SHS). It is also funded by the National Medical Research Council Centre Grant awarded to the Singapore General Hospital (CGAug16M011), National Innovation Challenge 
on Active and Confident Ageing (MOH/NIC/HAIG04/2017), National Research Foundation Cities of Tomorrow Award (COT-H1-2020-1) and the AM-ETHOS Duke-NUS Medical Student Fellowship (AM-ETHOS01/FY2020C2/09-A43) Grants. The grants funded the research staff, assessment equipment and on-site conduct of the trial, and the researchers were independent from funders.

\section{Competing interests None declared.}

Patient consent for publication Not applicable.

Ethics approval This study was approved by SingHealth Institutional Review Board $2018 / 2115$. Participants gave informed consent to participate in the study before taking part.

Provenance and peer review Not commissioned; externally peer reviewed.

Data availability statement № data are available. No additional data are available.

Supplemental material This content has been supplied by the author(s). It has not been vetted by BMJ Publishing Group Limited (BMJ) and may not have been peer-reviewed. Any opinions or recommendations discussed are solely those of the author(s) and are not endorsed by BMJ. BMJ disclaims all liability and responsibility arising from any reliance placed on the content. Where the content includes any translated material, BMJ does not warrant the accuracy and reliability of the translations (including but not limited to local regulations, clinical guidelines, terminology, drug names and drug dosages), and is not responsible for any error and/or omissions arising from translation and adaptation or otherwise.

Open access This is an open access article distributed in accordance with the Creative Commons Attribution Non Commercial (CC BY-NC 4.0) license, which permits others to distribute, remix, adapt, build upon this work non-commercially, and license their derivative works on different terms, provided the original work is properly cited, appropriate credit is given, any changes made indicated, and the use is non-commercial. See: http://creativecommons.org/licenses/by-nc/4.0/.

\section{ORCID iD}

Jia Qi Lee http://orcid.org/0000-0001-5260-0102

\section{REFERENCES}

1 Boutayeb A, Boutayeb S. The burden of non communicable diseases in developing countries. Int J Equity Health 2005;4:2.

2 Evans C. Malnutrition in the elderly: a multifactorial failure to thrive. Perm J 2005;9:38.

3 Singh A, Misra N. Loneliness, depression and sociability in old age. Ind Psychiatry J 2009;18:51.

4 World Health Organization. World report on ageing and health, 2015.

5 Wilberforce M, Challis D, Davies L, et al. Person-centredness in the care of older adults: a systematic review of questionnaire-based scales and their measurement properties. BMC Geriatr : 2016;16:63.

6 Inui TS. The need for an integrated biopsychosocial approach to research on successful aging. Ann Intern Med 2003;139:391.

7 Cesari M, Araujo de Carvalho I, Amuthavalli Thiyagarajan J, et al. Evidence for the domains supporting the construct of intrinsic capacity. J Gerontol A Biol Sci Med Sci 2018;73:1653-60.

8 Beard JR, Jotheeswaran AT, Cesari M, et al. The structure and predictive value of intrinsic capacity in a longitudinal study of ageing BMJ Open 2019;9:e026119-11.

9 Gonzalez-Bautista E, Andrieu S, Gutiérrez-Robledo LM, et al. In the quest of a standard index of intrinsic capacity. A critical literature review. J Nutr Health Aging 2020;24:959-65.

10 Carvalho AD I, Martin C, Cesari M, WHO CLINICAL CONSORTIUM ON HEALTHY AGEING. Operationalising the concept of intrinsic capacity in clinical settings, 2017. Available: https://www.who. int/ageing/health-systems/clinical-consortium/CCHA2017backgroundpaper-1.pdf

11 Nestola T, Orlandini L, Beard JR, et al. COVID-19 and intrinsic capacity. J Nutr Health Aging 2020;24:692-5.

12 Belloni G, Cesari M, Frailty CM. Frailty and intrinsic capacity: two distinct but related constructs. Front Med 2019;6:1-5.

13 Charles A, Buckinx F, Locquet M. Prediction of adverse outcomes in nursing home residents according to intrinsic capacity proposed by the world Health organization. Journals Gerontol Ser A 2019:1-6.

14 Ramírez-Vélez R, Correa-Bautista JE, García-Hermoso A, et al. Reference values for handgrip strength and their association with intrinsic capacity domains among older adults. J Cachexia Sarcopenia Muscle 2019;10:278-86.

15 Ma L, Chhetri JK, Zhang L, et al. Cross-sectional study examining the status of intrinsic capacity decline in community-dwelling older adults in China: prevalence, associated factors and implications for clinical care. BMJ Open 2021;11:e043062.

16 World Health Organization. The International classification of functioning, disability and health. . The International Classification of Functioning, Disability and Health, 2002: 1149. 1-22. http://www. who.int/classifications/icf/training/icfbeginnersguide.pdf

17 Baker PS, Bodner EV, Allman RM. Measuring life-space mobility in community-dwelling older adults. J Am Geriatr Soc 2003;51:1610-4.

18 Peel C, Sawyer Baker P, Roth DL, et al. Assessing mobility in older adults: the UAB study of aging Life-Space assessment. Phys Ther 2005;85:1008-19.

19 James BD, Boyle PA, Buchman AS, et al. Life space and risk of Alzheimer disease, mild cognitive impairment, and cognitive decline in old age. Am J Geriatr Psychiatry 2011;19:961-9.

20 Xue Q-L, Fried LP, Glass TA, et al. Life-space constriction, development of frailty, and the competing risk of mortality: the women's health and aging study I. Am J Epidemiol 2008;167:240-8.

21 Rantakokko M, Portegijs E, Viljanen A, et al. Changes in life-space mobility and quality of life among community-dwelling older people: a 2-year follow-up study. Qual Life Res 2016;25:1189-97.

22 Kennedy RE, Sawyer P, Williams CP, et al. Life-space mobility change predicts 6-month mortality. J Am Geriatr Soc 2017;65:833-8.

23 Shumway-Cook A, Patla A, Stewart A, et al. Environmental components of mobility disability in community-living older persons. J Am Geriatr Soc 2003;51:393-8.

24 Rantakokko M, Iwarsson S, Mänty M, et al. Perceived barriers in the outdoor environment and development of walking difficulties in older people. Age Ageing 2012;41:118-21.

25 Deshpande N, Metter EJ, Lauretani F, et al. Interpreting fear of falling in the elderly: what do we need to consider? J Geriatr Phys Ther 2009;32:91-6.

26 Delbaere K, Crombez G, Vanderstraeten G, et al. Fear-related avoidance of activities, falls and physical frailty. A prospective community-based cohort study. Age Ageing 2004;33:368-73.

$27 \mathrm{Ko} \mathrm{H}$, Park Y-H, Cho B, et al. Gender differences in health status, quality of life, and community service needs of older adults living alone. Arch Gerontol Geriatr 2019;83:239-45.

28 Caetano SC, Silva CMFP, Vettore MV. Gender differences in the association of perceived social support and social network with selfrated health status among older adults: a population-based study in Brazil. BMC Geriatr 2013;13:1-14.

29 Tay LB, Chua MP, Tay EL, et al. Multidomain geriatric screen and physical fitness assessment identify Prefrailty/Frailty and potentially modifiable risk factors in community-dwelling older adults. Ann Acad Med Singap 2019;48:171-80.

30 Guralnik JM, Simonsick EM, Ferrucci L. A short physical performance battery assessing lower extremity function: association with self-reported disability and prediction of mortality and nursing home admission. Journals Gerontol 1994.

31 Podsiadlo D, Richardson S. The timed 'Up \& Go': a test of basic functional mobility for frail elderly persons. J Am Geriatr Soc 1991;39:142-8.

32 Bohannon RW. Reference values for the five-repetition sit-to-stand test: a descriptive meta-analysis of data from elders, 2016. Available: http://dx.doi.org/102466/pms1031215-222

33 Greenberg SA. The geriatric depression scale (GDS) validation of a geriatric depression screening scale: a preliminary report. Best Pract Nurs Care to Older Adults 2019.

34 Herdman M, Gudex C, Lloyd A. Development and preliminary testing of the new five-level version of EQ-5D (EQ-5D-5L). Qual Life Res : 2011.

35 Sahadevan S, Lim PP, Tan NJ, et al. Diagnostic performance of two mental status tests in the older Chinese: influence of education and age on cut-off values. Int J Geriatr Psychiatry 2000;15:234-41.

36 Kohout FJ, Berkman LF, Evans DA, et al. Two shorter forms of the CES-D (center for epidemiological studies depression) depression symptoms index. J Aging Health 1993;5:179-93.

37 Kaiser MJ, Bauer JM, Ramsch C. Validation of the mini nutritional assessment short-form (MNA®-SF): a practical tool for identification of nutritional status. J Nutr Heal Aging 2009.

38 Sousa-Santos AR, Amaral TF. Differences in handgrip strength protocols to identify sarcopenia and frailty - a systematic review. BMC Geriatr 2017;17:238.

39 Sawyer P, Allman RM. Resilience in mobility in the context of chronic disease and aging: cross-sectional and prospective findings from the University of Alabama at Birmingham (UAB) study of aging. New Front Resilient Aging Life-Strengths Well-Being Late Life 2010:310-39.

40 Morley JE, Malmstrom TK, Miller DK. A simple frailty questionnaire (frail) predicts outcomes in middle aged African Americans. J Nutr Health Aging 2012;16:601-8. 
41 Mahoney F, Barthel DW. Functional evaluation ; the Barthel index. A simple index of the independence useful in scoring improvement in the rehabilitation of the chronically ill. Md State Med J 1965;14:61-5.

42 Lawton MP, Brody EM. Assessment of older people: selfmaintaining and instrumental activities of daily living. Gerontologist 1969;9:179-86.

43 Hooper D, Coughlan J, Mullen MR. Structural equation modelling: Guidelines for determining model fit. Electron. J Bus Res Methods 2008.

44 Ministry of Manpower, National Population and Talent Division, Strategy Group Prime PMO,, Singapore Department of Statistics,. Population in brief, 2020. Available: https://www.strategygroup. gov.sg/files/media-centre/publications/population-in-brief-2020.pdf [Accessed 2020].

45 Kennedy RE, Almutairi M, Williams CP, et al. Determination of the minimal important change in the Life-Space assessment. J Am Geriatr Soc 2019;67:565-9.

46 Portegijs $\mathrm{E}$, Rantakokko $\mathrm{M}$, Viljanen $\mathrm{A}$, et al. Is frailty associated with life-space mobility and perceived autonomy in participation outdoors? A longitudinal study. Age Ageing 2016;45:550-3.

47 Lu S, Liu Y, Guo Y, et al. Neighbourhood physical environment, intrinsic capacity, and 4-year late-life functional ability trajectories of low-income Chinese older population: a longitudinal study with the parallel process of latent growth curve modelling. EClinicalMedicine 2021;36:100927.
48 Portegijs E, Rantakokko M, Mikkola TM. Association between physical performance and sense of autonomy in outdoor activities and life-space mobility in community-dwelling older people. J Am Geriatr Soc 2014;62:621.

49 Sartori AC, Wadley VG, Clay OJ, et al. The relationship between cognitive function and life space: the potential role of personal control beliefs. Psychol Aging 2012;27:364-74.

50 Béland F, Julien D, Bier N, et al. Association between cognitive function and life-space mobility in older adults: results from the FRéLE longitudinal study. BMC Geriatr : 2018;18:227.

51 Silberschmidt S, Kumar A, Raji MM, et al. Life-space mobility and cognitive decline among Mexican Americans aged 75 years and older. J Am Geriatr Soc 2017;65:1514-20.

52 Langer EJ, Benevento A. Self-Induced dependence. J Pers Soc Psychol 1978.

53 Cui G-H, Yao Y-H, Xu R-F, et al. Cognitive impairment using education-based cutoff points for CMMSE scores in elderly Chinese people of agricultural and rural Shanghai China. Acta Neurol Scand 2011:124:361-7.

54 Ahadi S, Zhou W, Schüssler-Fiorenza Rose SM, et al. Personal aging markers and ageotypes revealed by deep longitudinal profiling. Nat Med 2020;26:83-90.

55 Ho SH, Tan DPS, Tan PJ, et al. The development and validation of a prototype mobility tracker for assessing the life space mobility and activity participation of older adults. BMC Geriatr 2020;20:251. 